\title{
Detection of Right-Sided Endocarditis in Children with Congenital Heart Disease by Two-Dimensional Echocardiography
}

\author{
Süheyla Özkutlu, M.D.,* Muhsin Saraçlar, M.D.,* \\ Sencan Özme, M.D., * A. Yüksel Bozer, M.D., ${ }^{* *}$ \\ Ilhan Pasaoglu, M.D. ${ }^{\dagger}$ Metin Demircin, M.D. ${ }^{\dagger}$ \\ Ahmet Hatipoglu, M.D., ${ }^{\dagger}$ and Alpay Çeliker ${ }^{\dagger \dagger}$
}

\section{Summary}

Right-sided endocarditis is rare in children. Since the clinical picture is nonspecific and the frequency of sterile blood cultures is high, the clinical and laboratory diagnosis is difficult. Recent reports suggest that echocardiography is a useful method to detect the presence of right-sided endocarditis.

We studied 8 patients with congenital heart disease and right-sided endocarditis detected by two-dimensional echocardiography. All the patients were shown to have one or more vegetations in the two-dimensional echocardiographic examination. The 4 patients who did not respond to antibiotic therapy underwent elective cardiac surgery. One of these patients additionally had recurrent emboli to the lungs. Another of these 4 patients died during surgery due to myocardial failure. Apart from these 4 cases, urgent surgical intervention had to be carried out in 2 cases because of very large vegetative masses. Surgical confirmation of the diagnosis was available in all 6 cases.

According to these results, we can conclude that two-dimensional echocardiography assumes a specific diagnostic role in cases with rightsided endocarditis.

\section{Additional Indexing Words:}

Right-sided endocarditis Congenital heart disease sional echocardiography Surgical-operation

Two-dimen-

From the Department of Cardiology, Hacettepe University, Institute of Child Health, Ankara, Turkey.

* Professor of Pediatrics and Pediatric Cardiologist, Hacettepe University, Institute of Child Health.

** Professor of Cardiovascular Surgery, Hacettepe University, Faculty of Medicine.

+ Associate Professor of Cardiovascular Surgery, Hacettepe University, Faculty of Medicine.

t† Pediatric Cardiologist, Hacettepe University, Institute of Child Health.

Address for reprints: Süheyla Ozkutlu, M.D., Department of Pediatric Cardiology, Hacettepe University, Ankara, Turkey.

Received for publication June 22, 1989.

Accepted July 21, 1989. 
T $\mathrm{N}$ children right-sided endocarditis is rare ${ }^{11}$ and usually associated with Isolated pulmonary stenosis, patent ductus arteriosus, tetralogy of Fallot and ventricular septal defect. ${ }^{2)}$ Since the clinical picture is nonspecific and the frequency of sterile cultures is high, the clinical and laboratory diagnoses are difficult." Successful management of infective endocarditis depends on early recognition, although nonspecific presentation and negative cultures may delay confirmation of the diagnosis. Recent reports suggest that echocardiography is a useful method to detect the presence of right-sided endocarditis. ${ }^{4)-12)}$ There are few children in whom echocardiographic demonstration of the right-sided endocarditis has been reported. ${ }^{12)}$ This report describes our clinical and echocardiographic experience with right-sided infective endocarditis.

\section{Subjects and Methods}

During the last 5 years we studied 8 patients with congenital heart disease and right-sided endocarditis. There were 6 male and 2 female patients ranging in age from 4 to 12 years.

In addition to routine investigations and cardiac catheterizations, twodimensional, M-mode, Doppler and peripheral vein contrast studies were performed using a Toshiba SSH-60A echocardiograph equipped with 3.75 and $2.5 \mathrm{MHz}$ transducers. The images were recorded on video tape and Polaroid film. As contrast material 3\% saline was used as in a method described previously. ${ }^{13)}$

All cardiac structures were specifically examined for evidence of vegetations.

\section{Results}

The clinical and investigative data of the patients are summarized in Table I. All 8 patients had congenital cardiac lesions proved by cardiac catheterization and angiocardiography. Four (cases 2, 4,6, 7) had previously undergone corrective cardiac surgery and had residual ventricular septal defects as confirmed by Doppler echocardiography.

Two-dimensional and Doppler echocardiography was performed in every patient during the acute illness. Furthermore, peripheral vein contrast echocardiography was used in 4 patients with residual ventricular septal defects. Passagc of the contrast material from the RV to LV was secn. In all patients, the previous diagnoses of the congenital cardiac lesions were confirmed. In addition, all the patients were shown to have one or more vegetations on 
Vol. 31
No. 2

2-D ECHO IN RIGHT-SIDED ENDOCARDITIS

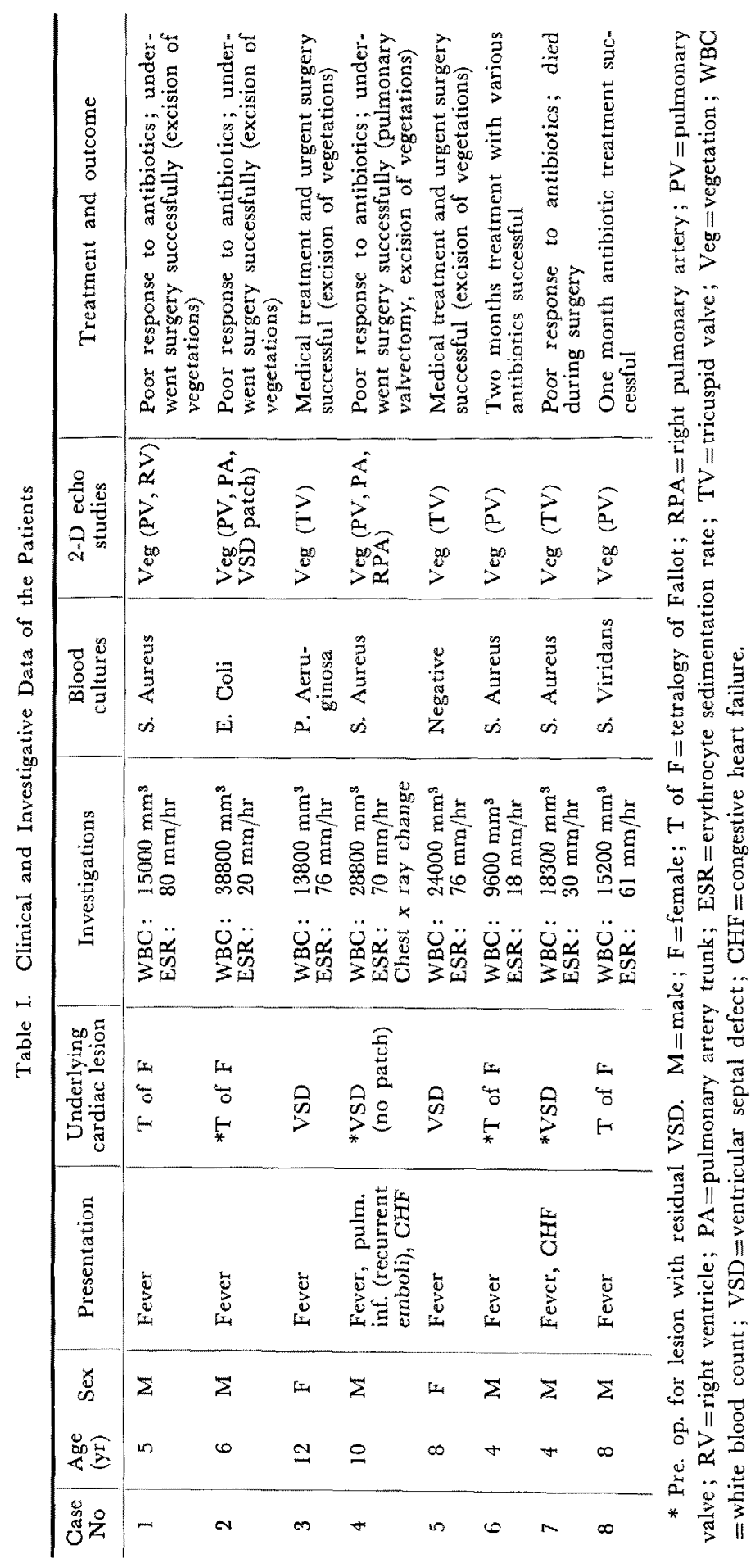


the echocardiographic examination. The vegetations were situated on the pulmonary valve and in the ventricular cavity in case I (Figs. 1, 2); on the pulmonary valve in cases 6 and 8; on the pulmonary valve, in the pulmonary artery and on the ventricular septal defect patch in case 2 (Fig. 3); on the septal leaflet of the tricuspid valve in cases 3, 5 and 7 (Fig. 4); and on the pulmonary valve, in the entire pulmonary trunk as well as in the right pulmonary branch in case 4 (Fig. 5).
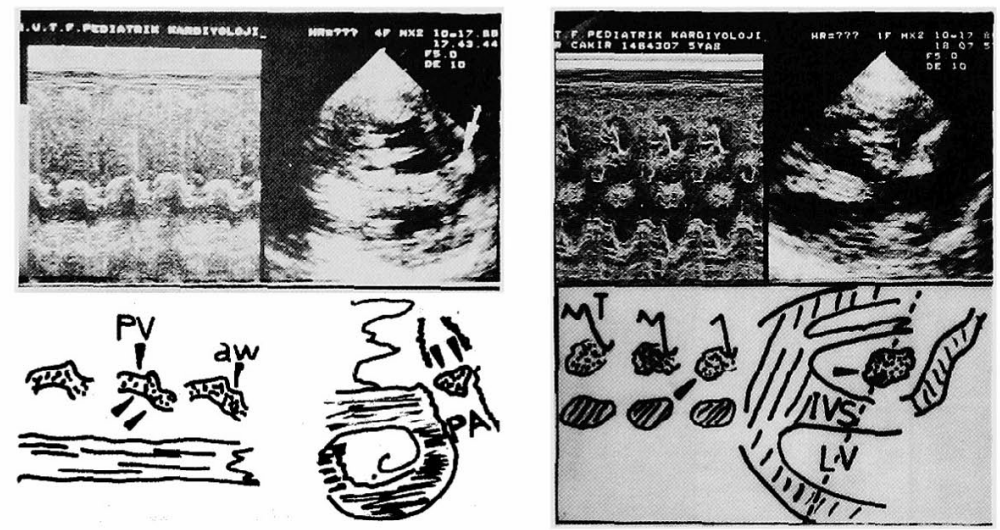

Fig. 1. M-mode echocardiogram (left) showing thickened pulmonary valve and "multi-layered" echoes (arrows). Two-dimensional echocardiogram (right) showing the vegetation on the pulmonary valve (arrows). PV= pulmonary valve; $\mathrm{PA}=$ pulmonary artery; $\mathrm{aw}=\mathrm{a}$ wave.

Fig. 2. M-mode (left) and two-dimensional echocardiograms (right) showing an echo-dense mass suggesting a vegetation (arrowhead) in the right ventricle. At surgery a vegetation $0.5 \times 1.5 \mathrm{~cm}$ in diameter was found on the supraventricular crest. $T=$ tricuspid valve; $L V=$ left ventricle; IVS $=$ interventricular septum.

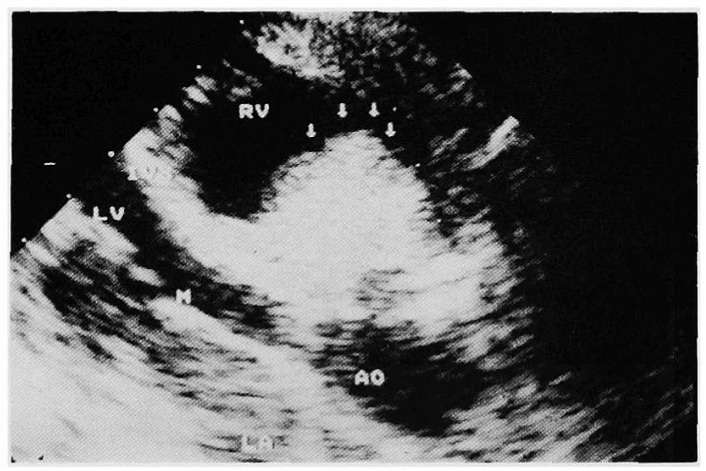

Fig. 3. Two-dimensional echocardiogram in the parasternal long-axis view showing a large vegetation (arrows) on the VSD patch. LV=left ventricle; IVS=interventricular septum; $\mathrm{RV}=$ right ventricle; $\mathrm{M}=$ mitral valve; $\mathrm{LA}=$ left atrium; $\mathrm{AO}=$ aorta. 


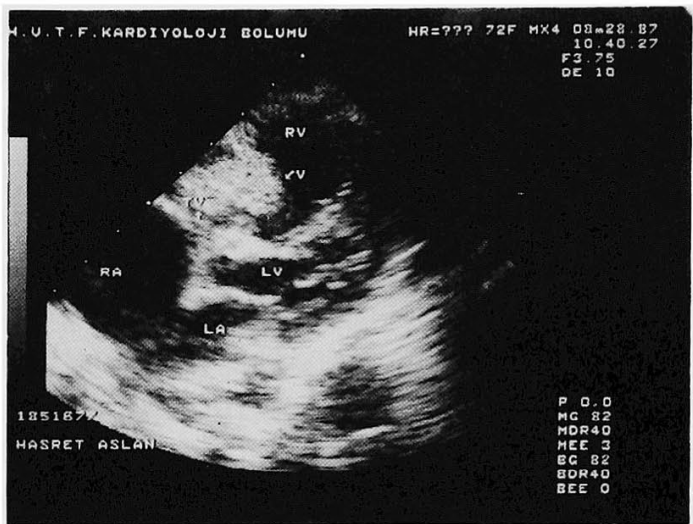

Fig. 4. Two-dimensional echocardiogram from a subcostal view showing a very large vegetation (arrows) on the septal leaflet of the tricuspid valve. $R V=$ right ventricle; $V=$ vegetation; $T V=$ tricuspid valve; $L V=$ left ventricle; $\mathrm{RA}=$ right atrium; $\mathrm{LA}=$ left atrium.

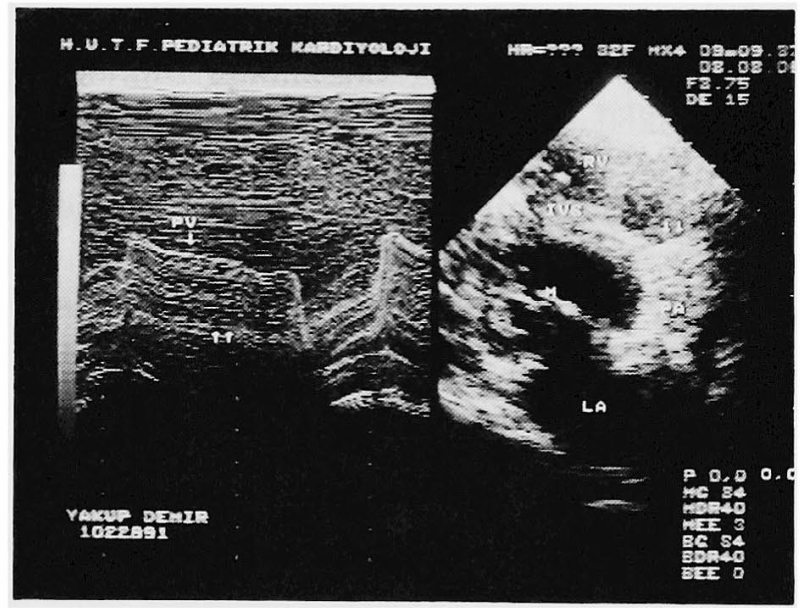

Fig. 5. M-mode echocardiogram showing "multi-layered" echo-dense image of the pulmonary valve during diastole. Two-dimensional echocardiogram shows a massive vegetation (arrows) occupying the entire pulmonary artery. $\mathrm{PV}=$ pulmonary valve; $\mathrm{RV}=$ right ventricle; IVS = interventricular septum; $\mathbf{M}=$ mitral valve $\mathbf{L A}=$ left atrium; $\mathrm{PA}=$ pulmonary artery.

The M-mode echocardiogram demonstrated that vegetations on the pulmonary valve appeared as " multi-layered" echo-dense masses or thickening of the pulmonary valve during diastole and/or systole, and as "shaggy" irregular echo masses attached to the tricuspid valve without restricting its motion. Two-dimensional echocardiography enabled direct visualization of vegetative masses. All vegetative masses were larger than $5 \mathrm{~mm}$ in diameter at the time the patients were admitted to the hospital. 
Blood cultures were positive in all but one of the cases (case 5) in whom the diagnosis was confirmed at operation. Staphylococcus aureus was isolated in cases 1, 4, 6 and 7, Streptococcus viridans in case 8 and Escherichia coli and Pseudomonas aeruginosa in cases 2 and 3, respectively.

The 4 patients who did not respond to antibiotic therapy (cases 1, 2, 4 and 7) underwent elective cardiac surgery. At operation vegetations were seen on the supraventricular crest and on the pulmonary valve in case 1 . In case 2 vegetations were on the pulmonary valve, in the main pulmonary artery and on the ventricular septal defect patch. All of these vegetations were surgically removed. In addition, the ventricular septal defects were closed. Case 7 died during surgery due to myocardial failure. Because of uncontrolled sepsis and recurrent pulmonary emboli, case 4 required cardiac surgery. It was successfully performed. At surgery vegetations were seen on the pulmonary valve, in the entire pulmonary trunk and in the right pulmonary artery. In this case pulmonary valvectomy was done, vegetations were excised and the ventricular septal defect was closed. Urgent surgical intervention was carried out in cases 3 and 5 because of very large vegetative masses. Their ventricular septal defects were closed and vegetations on the septal leaflet of the tricuspid valve were excised.

Histologic examinations of the surgically excised tissues in all cases revealed findings consistent with an infective vegetation.

The other 2 patients (cases 6 and 8 ) were treated medically.

\section{Drscussion}

Involvement of the right side of the heart occurs in about 5 to $10 \%$ of patients with infective endocarditis. ${ }^{7}$ Tricuspid valve endocarditis is more common than that of the pulmonary valve." In recent years, the incidence of right-sided endocarditis has increased dramatically, especially among narcotic addicts. ${ }^{4), 6), 10), 14)-16)}$ In children, right-sided endocarditis is uncommon ${ }^{11}$ and usually occurs in patients with congenital heart disease, mostly in cases of isolated pulmonary stenosis, patent ductus arteriosus, tetralogy of Fallot and ventricular septal defect. ${ }^{2}$ ) In the review by Johnson et al on subacute bacterial endocarditis in children, there were 30 autopsy cases. ${ }^{2}{ }^{2}$ In this report, vegetations were present in the left side of the heart in 20 cases and in the right side in 7 cases. The pulmonary valve and/or pulmonary artery was involved in 4 and the tricuspid valve in 3 . During the last 5 years, 29 patients with congenital heart disease and infective endocarditis were followed up in our institution, with 8 having right-sided endocarditis. Both the findings of Johnson et al and ours suggest that right-sided endocarditis is not 
a rare type of this disease.

The clinical picture of right-sided endocarditis is usually dominated by pulmonary manifestations and therefore correct diagnosis cannot be suspected by clinical findings alone. ${ }^{17,7), 12)}$ In the report by Ghia et al the clinical manifestations of $\mathbf{8}$ cases with right-sided endocarditis were mainly fever, recurrent pneumonia and respiratory complaints. ${ }^{12)}$ In our cases fever was the most common symptom. One was admitted to the hospital with pneumonia and right-sided congestive heart failure (case 4). These results emphasize that signs traditionally associated with right-sided endocarditis are too uncommon to be helpful in early diagnosis.

Recent reports suggest that cross-sectional echocardiography is often useful in demonstrating the presence and position of infective vegetations. But the exact sensitivity of this technique is unknown. In some reports, tricuspid valve vegetations were seen in 80 to $100 \%$ of patients with tricuspid valve endocarditis utilizing two-dimensional echo and in 20 to $65 \%$ with $\mathrm{M}$-mode echocardiography. ${ }^{71}$ In 1986, Robbins et al ${ }^{10)}$ reported 21 patients presenting with 23 episodes of right-sided endocarditis. Eighteen patients gave a history of intravenous drug abuse and only one patient had preexisting congenital heart disease. Echocardiographic examination showed a vegetation in 19 of 23 episodes. The vegetation was sited on the tricuspid valve in 14 episodes $(70 \%)$, on the pulmonary valve in 3 episodes $(16 \%)$, and on both the tricuspid valve and the mitral valve and on both the tricuspid valve and the aortic valve in each of the remaining 2 episodes, respectively (5\%). In 1988 Chia et al reported 8 patients with right-sided endocarditis. ${ }^{\mathbf{1 2}}$ All 8 patients whose vegetations were diagnosed by two-dimensional echocardiography had congenital cardiac lesions. The vegetations in 7 of their cases $(88 \%)$ were present either in the body of the right ventricle, in the pulmonary valve, or in the main pulmonary artery. In only one case $(13 \%)$ was the tricuspid valve involved. This report and our study have shown that echocardiography is a very valuable noninvasive method not only for the early diagnosis of this disease but also for determination of the site of the vegetations. Although the tricuspid valve is the most frequently involved valve among intravenous drug abusers, ${ }^{7), 10), 14)-16)}$ in congenital heart discase the pulmonary valve and artery are the most frequently involved sites as shown in the present study and by others. ${ }^{51,81,9), 111,12)}$

Karl et al reported that vegetations must be larger than 2-3 $\mathrm{mm}$ in diameter to be visualized by current echocardiographic techniques. ${ }^{17}$ ) In the present study all patients had vegetations larger than $5 \mathrm{~mm}$ in diameter at the time they were admitted to the hospital. In 3 of our patients (cases 1, $2,4)$, the size of the vegetations increased during antibiotic therapy and 
surgical intervention had to be carried out.

It can be concluded that echocardiography assumes a specific diagnostic role in cases with right-sided endocarditis and echocardiography of the right heart should be performed in all cases of congenital malformations presenting with fever and respiratory complaints to detect unsuspected instances of endocarditis.

\section{RefERENCES}

1. Anderson RH, MaCartney FJ, Shinebourne EA, Tynan M (eds): Infective endocarditis. in Paediatric Cardiology, Churchill Livingstone, London, p 1229, 1987

2. Johnson DH, Rosenthal A, Nadas AS: A forty-year review of bacterial endocarditis in infancy and childhood. Circulation 51:581, 1975

3. Sharma S, Desai AG, Pillai MG, Kinare SG, Panday SR: Clinical and diagnostic features of pulmonary valve endocarditis in the setting of congenital cardiac malformations. Int $\mathrm{J}$ Cardiol 9: 457, 1985

4. Mehlman DJ, Furey W, Phair J, Eftron M, Hartz R: Two-dimensional echocardiographic features diagnostic of isolated pulmonic valve endocarditis. Am Heart J 103: 137, 1982

5. Dander B, Righetti B, Poppi A: Echocardiographic diagnosis of isolated pulmonary valve endocarditis. Br Heart J 47: 298, 1982

6. Berger M, Wilkes HS, Gallerstein PE, Berdoft RL, Goldberg E: M-mode and two-dimensional echocardiographic findings in pulmonic valve endocarditis. Am Heart $\mathrm{J}$ 107: 391, 1984

7. Panidis IP, Kotler MN, Mintz GS, Segal BL, Ross JJ: Right heart endocarditis: Clinical and echocardiographic features. Am Heart J 107: 759, 1984

8. Vargas-Barron J, Attie F, Buendia-Hernandez A, Keirn C, Esquivel-Avila J: Echocardiographic recognition of pulmonary artery endarteritis in patent ductus arteriosus. Am Heart $J$ 109: 368,1985

9. Chambers J, Leech G: Echocardiographic demonstration of pulmonary valve endocarditis associated with congenital ventricular septal defect. Br Heart J 55: 494, 1986

10. Robbins M, Frater R, Soeiro R, Frishman W, Strom J: Influence of vegetation size on clinical outcome of right-sided infective endocarditis. Am J Med 80: 165, 1986

11. Chia BL, Ee BK, Choo MH, Tan NG: Two-dimensional echocardiographic features of pulmonary artery vegetation. Am Heart J 113: 833, 1987

12. Chia BL, Yan PG, Ee BK, Choo MH, Lee CN, Tay MB: Right-sided infective endocarditis: A Singapore experience. Am Heart J 116: 568, 1988

13. Saraçlar M, Özme S, Bilgiç A, Özkutlu S, Öztunç F: Two-dimensional contrast echocardiography in atrial septal defect. Abstracts of Scientific Presentations, XVIII International Congress of Pediatrics, Honolulu, Hawaii, July 7-12, p 393, 1986

14. Roberts WC, Buchbinder NA: Right-sided valvular infective endocarditis: A clinicopathologic study of twelve necropsy patients. Am J Med 53: 7, 1972

15. Banks $T$, Fletcher R, Ali N: Infective endocarditis in heroin addicts. Am J Med 55: 444, 1973

16. Berger M, Delfin LA, Jelveh M, Goldberg E: Two-dimensional echocardiographic findings in right-sided infective endocarditis. Circulation 61: 855, 1980

17. Karl T, Wensley D, Stark J, Leval M, Rees P, Taylor JFN: Infective endocarditis in children with congenital heart disease: comparison of selected features in patients with surgical correction or palliation and those without. Br Heart J 58: 57, 1987 